\title{
Prevalência dos achados endoscópicos em Sergipe
}

\author{
Prevalence of endoscopic findlings in Sergipe \\ Prevalencia de hallazgos endoscópicos en Sergipe
}

Recebido: 12/10/2021 | Revisado: 19/10/2021 | Aceito: 20/10/2021 | Publicado: 21/10/2021

Rinaldo Alves da Silva Rolim Júnior
ORCID: https://orcid.org/0000-0003-4702-5320
Universidade Tiradentes, Brasil
E-mail:rolimrinaldo@gmail.com
Amanda Santos Meneses Barreto
ORCID: https://orcid.org/0000-0001-5449-8180
Universidade Tiradentes, Brasil
E-mail: amandabarreto.ms@gmail.com
Elisandra de Carvalho Nascimento
ORCID: https://orcid.org/0000-0002-1309-5622
Universidade Tiradentes, Brasil
E-mail: elis.carvalhonascimento@gmail.com
Marília de Lima Mota
ORCID: https://orcid.org/0000-0002-5620-4569
Universidade Tiradentes, Brasil
E-mail: mariliadelimamota@gmail.com
Frederico Santana de Lima
ORCID: https://orcid.org/0000-0002-5251-5976
Universidade Tiradentes, Brasil
E-mail: fredericogastro@gmail.com
Ana Celia Goes Melo Soares

\section{Resumo}

Objetivo: Descrever o perfil epidemiológicos dos pacientes submetidos a endoscopia digestiva alta (EDA) em Sergipe. Métodos: Trata-se um estudo retrospectivo a partir de laudos de pacientes submetidos a endoscopia digestiva alta em duas clínicas particulares de Aracaju. Foram analisados dados dos prontuários informatizados de pacientes que realizaram EDA entre os anos de 2015 e 2019. Resultados: Do total de 1199 laudos de EDA, 11 exames (0,91\%) não mostraram quaisquer lesões patológicas. A média de idade foi de 42,7 anos com predominância do sexo feminino $(64,47 \%)$. Os órgãos de patologias mais prevalentes foram o estômago (64,2\%), seguido de esôfago (24,5\%) e duodeno $(11,3 \%)$. A esofagite não erosiva e esofagite erosiva foram as patologias mais comuns do esôfago. Gastrite foi a patologia mais prevalente no estômago e a linfagiectasia idiopática a mais comum do duodeno. Em pacientes que realizaram bariátrica obteve maior incidência esofagite não erosiva e pacientes que realizaram cirurgia antirrefluxo apresentaram gastrite moderada com maio prevalência. Conclusão: De acordo com o estudo, foi visto que a maioria da amostra foi composta por mulheres, sendo mais da metade das patologias encontradas foram no estômago. Entretanto, na literatura há divergências e convergências acerca de patologias, gênero e faixa etária mais prevalentes devido a diferentes características sociodemográficas. Dessa forma, são necessários maiores estudos a fim de estabelecer melhores indicativos das variáveis sociodemográficas.

Palavras-chave: Endoscopia; Esôfago; Estomago; Duodeno.

\begin{abstract}
Objective: To describe the epidemiological profile of patients undergoing upper digestive endoscopy (EDA) in Sergipe. Methods: This is a retrospective study based on reports of patients undergoing upper digestive endoscopy in two private clinics in Aracaju. Data from computerized medical records of patients who underwent EDA between the years 2015 and 2019 were analyzed. Results: Of the total of 1199 EDA reports, 11 exams $(0.91 \%)$ did not show any pathological lesions. The average age was 42.7 years, with a predominance of females $(64.47 \%)$. The most prevalent pathological organs were the stomach $(64.2 \%)$, followed by the esophagus $(24.5 \%)$ and duodenum $(11.3 \%)$. Non-erosive esophagitis and erosive esophagitis were the most common pathologies of the esophagus. Gastritis was the most prevalent pathology in the stomach and idiopathic lymphagiectasia was the most common in the duodenum. In patients who underwent bariatric had a higher incidence of non-erosive esophagitis and patients who underwent anti-reflux surgery had moderate gastritis with higher prevalence. Conclusion: According to the study, it was seen that the majority of the sample was composed of women, with more than half of the pathologies found in the stomach. However, in the literature there are divergences and convergences about the most prevalent pathologies, gender and age group due to
\end{abstract}


different sociodemographic characteristics. Thus, further studies are needed in order to establish better indicators of sociodemographic variables.

Keywords: Endoscopy; Esophagus; Stomach; Duodenum.

\begin{abstract}
Resumen
Objetivo: Describir el perfil epidemiológico de los pacientes sometidos a endoscopia digestiva alta (EDA) en Sergipe. Métodos: Este es un estudio retrospectivo basado en informes de pacientes sometidos a endoscopia digestiva alta en dos clínicas privadas en Aracaju. Se analizaron los datos de las historias clínicas informatizadas de los pacientes sometidos a EDA entre los años 2015 y 2019. Resultados: Del total de 1199 informes EDA, 11 exámenes (0,91\%) no mostraron lesiones patológicas. La edad promedio fue de 42,7 años, con predominio del sexo femenino (64,47\%). Los órganos patológicos más prevalentes fueron el estómago (64,2\%), seguido del esófago $(24,5 \%)$ y el duodeno (11,3\%). La esofagitis no erosiva y la esofagitis erosiva fueron las patologías más frecuentes del esófago. La gastritis fue la patología más prevalente en el estómago y la linfagiectasia idiopática fue la más común en el duodeno. En los pacientes que se sometieron a cirugía bariátrica tuvo mayor incidencia de esofagitis no erosiva y los pacientes que se sometieron a cirugía antirreflujo presentaron gastritis moderada con mayor prevalencia. Conclusión: Según el estudio, se observó que la mayoría de la muestra estaba compuesta por mujeres, con más de la mitad de las patologías encontradas en el estómago. Sin embargo, en la literatura existen divergencias y convergencias sobre las patologías más prevalentes, género y grupo de edad debido a diferentes características sociodemográficas. Por tanto, se necesitan más estudios para establecer mejores indicadores de las variables sociodemográficas.
\end{abstract}

Palabras clave: Endoscopia; Esófago; Estómago; Duodeno.

\title{
1. Introdução
}

A endoscopia digestiva alta (EDA) tornou-se um exame fundamental para diagnósticos de patologias que acometem o trato digestivo superior, como doença ulcerosa péptica, esôfago de Barret, neoplasias gástricas e outras doenças que acometem este trato e precisam de biópsia para confirmação do diagnóstico. Apesar de poder ser utilizada para terapêutica, a grande importância da endoscopia digestiva alta (EDA) se deve a capacidade de estabelecer diagnósticos, sobretudo precoces, seja em pacientes sintomáticos ou até mesmo naqueles que não possuem nenhuma queixa gastrointestinal. Assim, caso exista alguma lesão advinda do trato gastrointestinal superior, sua incidência pode ser bastante reduzida desde que haja uma triagem e um diagnóstico precoce a partir da endoscopia (Shaukat et al., 2015)

Atualmente, o diagnóstico da maioria das afecções do estômago é realizado pela histopatologia através da análise e classificação de biópsias coletadas pela EDA (Ribeiro, Coelho, \& Coelho, 2019). A gastrite possui diversos fatores etiológicos e pode ser classificada de acordo com sua evolução temporal, em aguda e crônica, seu mecanismo patogênico, a localização anatômica e características histológicas (MATA et al., 2016). No que se refere aos tumores do trato gastrointestinal, mais de um milhão de casos de câncer gástrico são diagnosticados a cada ano em todo o mundo, sendo o câncer de estômago o quinto mais comumente diagnosticado no mundo e o sétimo mais prevalente (Rawla\&Barsouk, 2019).

Diante do que foi explanado, é incontestável que os achados endoscópicos do trato gastrointestinal superior representam um problema de saúde pública, uma vez que se correlacionam diretamente a uma necessidade de consultas médicas, exames, acompanhamento em longo prazo da lesão, hospitalizações, cirurgias e óbitos (Zagari et al., 2016).

Até o momento, no Brasil, observa-se uma escassez de estudos que apresentem dados sobre os principais diagnósticos observados nas diferentes faixas etárias por meio da EDA. Assim, torna-se necessária a elaboração de estudos sistematizados e bem conduzidos para definição da realidade local acerca da prevalência dos achados endoscópicos, objetivando a orientação de condutas médicas individuais e institucionais, principalmente no que diz respeito à propedêutica e a terapêutica das doenças do trato digestivo superior (Durães, Fabris, Faraco, Madeira, \& Luca, 2010). Este conhecimento proporciona embasamento para o desenvolvimento de intervenções fundamentais na prevenção de lesões do trato gastrointestinal superior, a fim de evitar complicações futuras, determinando o importante papel da epidemiologia no controle da doença e, sobretudo, na melhoria da saúde da população (RAMOS et al., 2016). 
O levantamento de informações acerca dos achados na EDA tem ainda importância científica, uma vez que a existência de dados sobre esses achados relativos à Sergipe propicia o surgimento de novas indagações que podem impulsionar futuros estudos e trabalhos científicos. Dessa forma, o objetivo do presente estudo é descrever o perfil epidemiológico dos pacientes submetidos à endoscopia digestiva alta (EDA) no estado de Sergipe.

\title{
2. Metodologia
}

\section{Delineamento metodológico}

Trata-se de um estudo descritivo, quantitativo, retrospectivo, transversal a partir de laudos de pacientes submetidos a endoscopia digestiva alta em duas clínicas particulares de Aracaju.

\begin{abstract}
Amostra
A população deste estudo foi composta laudos endoscópicos encaminhados por médicos para clinicas de dois serviços particulares na cidade de Aracaju/SE a fim de realizar uma endoscopia digestiva alta (EDA). Foram analisados dados dos prontuários informatizados de pacientes que realizaram EDA entre os anos de 2015 e 2019. Foi realizado um censo de todos os prontuários dos pacientes, totalizando 1200 laudos. É critério de exclusão os prontuários com insuficiência de dados e menores de 18 anos.
\end{abstract}

\section{Coleta de dados}

A coleta de dados foi realizada a partir da seleção de todos os laudos endoscópicos informatizados de pacientes que preencheram todos os critérios de inclusão e nenhum critério de exclusão deste estudo. Os dados foram coletados pelo pesquisador nas clínicas duas clínicas particulares. Foi realizada a criação de um sistema de coleta de dados contendo as variáveis do estudo: sexo, idade, e os achados endoscópicos.

\section{Confecção do banco de dados e análise dos resultados}

Os dados coletados foram inseridos em uma planilha do Excel e posteriormente analisados no software R Studio versão 3.4.2. Estes dados serão analisados descritivamente por intermédio de frequências absolutas e relativas.

\section{Aspectos éticos}

Não haverá qualquer tipo de desconforto e/ou risco para os pacientes em questão e nenhum tipo de benefício para os mesmos está previsto. Os benefícios oriundos da presente pesquisa se aplicam a grupos posteriores de pacientes. Dados pessoais dos pacientes não serão abordados ou publicados. Quanto ao Termo de Consentimento Livre e Esclarecido (TCLE) dos pacientes, este não se aplica a esta pesquisa, uma vez que se trata de estudo retrospectivo, e os laudos são de guarda da clínica.

O projeto do estudo foi apresentado ao Comitê de Ética e Pesquisa da Universidade Tiradentes, via Plataforma Brasil, visando atender às recomendações da resolução no 466/2012 do Conselho Nacional de Saúde. Possui aprovação do CEP/UNIT sob o número 3.390.887.

\section{Resultados}

Foram analisados, retrospectivamente, uma amostra total de 1199 laudos de EDA sendo que 11 exames $(0,91 \%)$ não mostraram quaisquer lesões patológicas, sendo assim, consideradas com resultado normal. No que se refere a média de idade 
foi de 42,7 anos, com intervalo compreendendo a idade de 18 anos e 77 anos. Com relação à predominância do sexo, foi visto que a mostra foi composta por do sexo feminino $821(68,47 \%)$ e 378 (31,52\%) do sexo masculino. (Gráfico 1).

Gráfico 1: Proporção entre homens e mulheres.

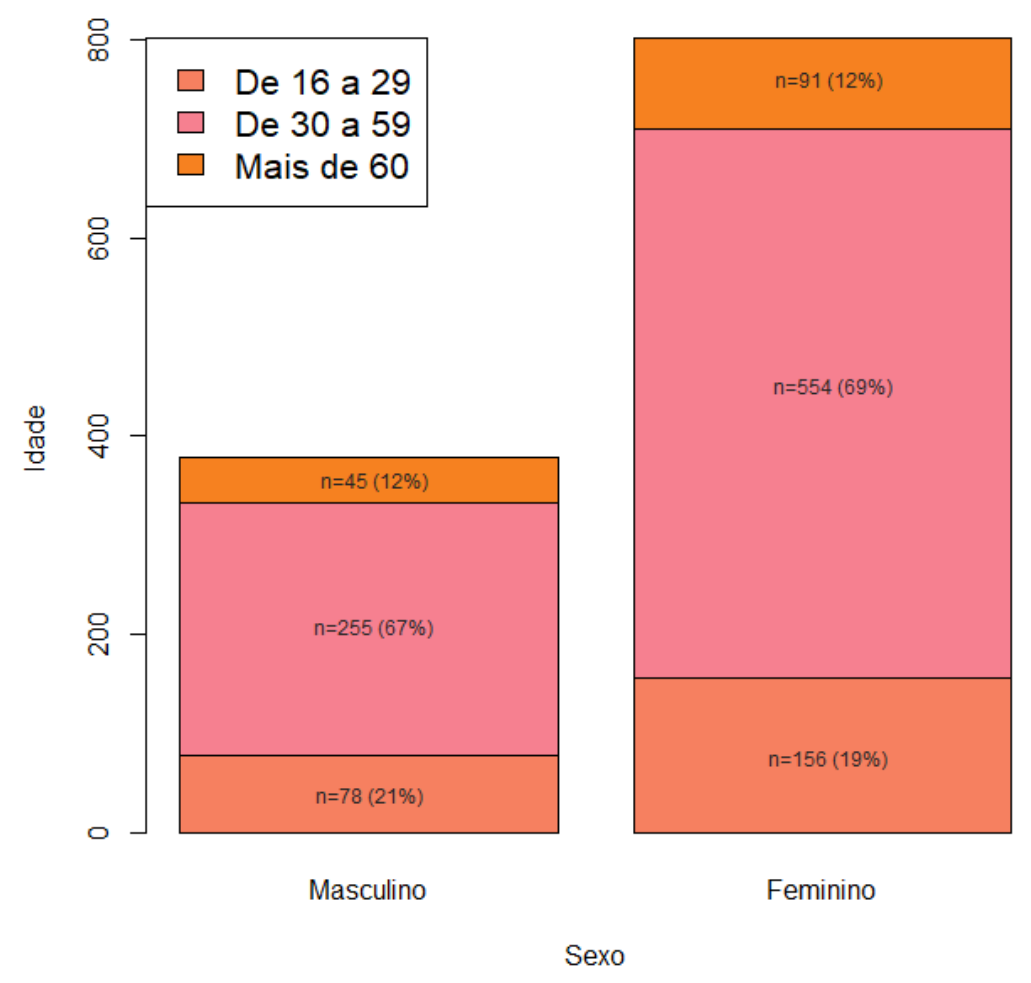

Fonte: Elaborado pelos autores.

Para o presente estudo, foi realizada uma divisão dos principais diagnósticos ou achados endoscópicos por órgãos, esôfago, estômago e duodeno. Dentre esses seguimentos do trato gastrintestinal, foi visto que $64,2 \%$ dos laudos apresentaram alguma patologia ou alteração no estômago e em 11,3\% no duodeno. (Gráfico 2). 
Gráfico 2: Proporção de patologias encontradas por órgãos do trato gastrintestinal alto.

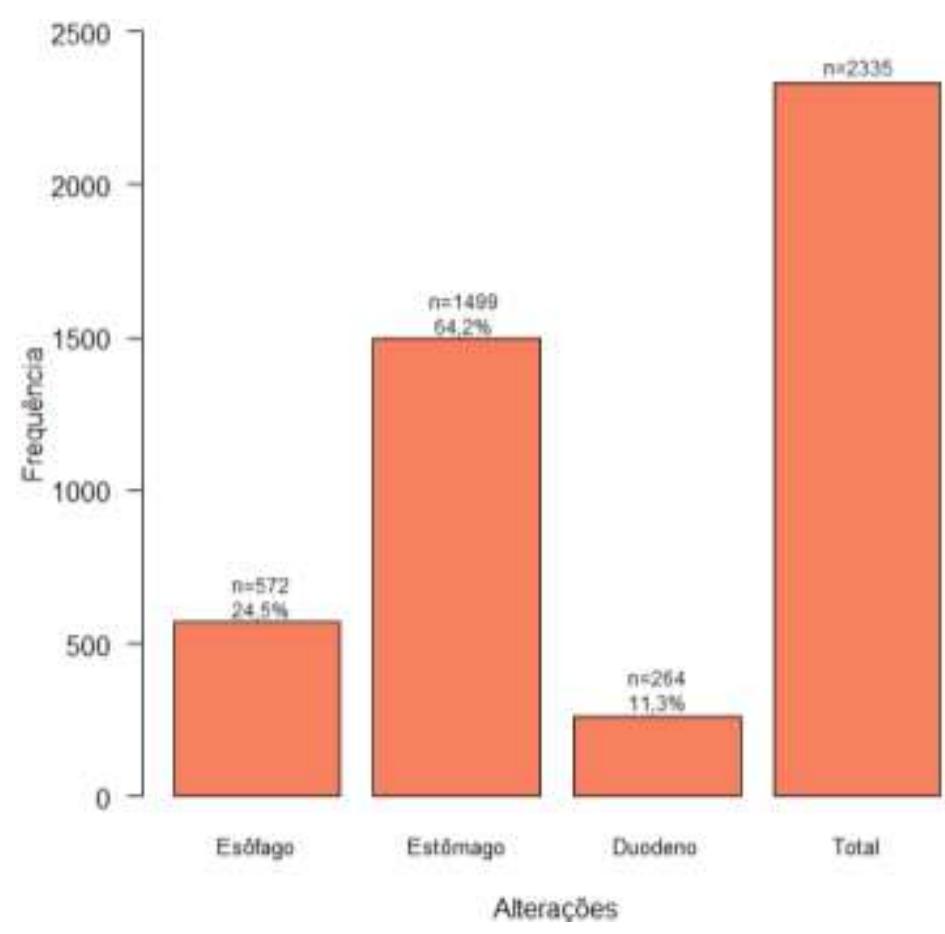

Fonte: Elaborado pelos autores.

Acerca dos diagnósticos encontrados no esôfago, foi visto que 29,77\% dos laudos apresentavam algum tipo de esofagite, sendo a patologia mais encontrada em ambos os sexos. Das esofagites encontradas, a esofagite não erosiva foi vista em 143 (11,92\%) laudos e a esofagite grau A na classificação de Fulano em 141 laudos (11,75\%). Relativo à idade, foi analisado que a idade mais acometida com essa patologia estava na faixa dos 30 aos 59 anos. (Tabela 1, Tabela 2 e Tabela 3). 
Research, Society and Development, v. 10, n. 13, e567101321705, 2021

(CC BY 4.0) | ISSN 2525-3409 | DOI: http://dx.doi.org/10.33448/rsd-v10i13.21705

Tabela 1: Quantidade de alterações - Esôfago

\begin{tabular}{|c|c|c|}
\hline Alteração & $\mathbf{N}$ & $\%$ \\
\hline Esofagite inespecífica & 4 & $1 \%$ \\
\hline Esofagite não erosiva & 143 & $25 \%$ \\
\hline Esofagite grau A & 141 & $25 \%$ \\
\hline Esofagite grau B & 61 & $11 \%$ \\
\hline Esofagite grau C & 7 & $1 \%$ \\
\hline Esofagite grau D & 5 & $1 \%$ \\
\hline Esofagite eosinofílica & 3 & $1 \%$ \\
\hline Megaesôfago & 5 & $1 \%$ \\
\hline Neoplasia de esôfago & - & - \\
\hline Monilíase grau I & 19 & $3 \%$ \\
\hline Monilíase grau II & 1 & $0 \%$ \\
\hline Monilíase grau III & - & - \\
\hline Monilíase grau IV & - & - \\
\hline Hérnia de hiato & 93 & $16 \%$ \\
\hline Esôfago de barret curto & 22 & $4 \%$ \\
\hline Esôfago de barret longo & 7 & $1 \%$ \\
\hline Varizes & 7 & $1 \%$ \\
\hline Estenose & 3 & $1 \%$ \\
\hline Úlcera & 6 & $1 \%$ \\
\hline Xantelasma & 5 & $1 \%$ \\
\hline Nódulo submucoso & 1 & $0 \%$ \\
\hline Lesão elevada no esôfago & 39 & $7 \%$ \\
\hline Total & 572 & $100 \%$ \\
\hline
\end{tabular}

Fonte: Elaborado pelos autores. 
Tabela 2: Quantidade de alterações por sexo - Esôfago

\begin{tabular}{|c|c|c|c|}
\hline Alteração & Masculino & $\%$ & Feminino \\
\hline Esofagite inespecífica & - & - & 4 \\
\hline Esofagite não erosiva & 37 & $6 \%$ & 106 \\
\hline Esofagite grau A & 52 & $9 \%$ & 89 \\
\hline Esofagite grau B & 40 & $7 \%$ & 21 \\
\hline Esofagite grau $\mathrm{C}$ & 6 & $1 \%$ & 1 \\
\hline Esofagite grau D & 3 & $1 \%$ & 2 \\
\hline Esofagite eosinofílica & 3 & $1 \%$ & - \\
\hline Megaesôfago & - & - & 5 \\
\hline Neoplasia de esôfago & - & - & - \\
\hline Monilíase esofágica grau I & 10 & $2 \%$ & 9 \\
\hline Monilíase esofágica grau II & - & - & 1 \\
\hline Monilíase esofágica grau III & - & - & - \\
\hline Monilíase esofágica grau IV & - & - & - \\
\hline Hérnia de hiato & 28 & $5 \%$ & 65 \\
\hline Esôfago de barret curto & 13 & $2 \%$ & 9 \\
\hline Esôfago de barret longo & 3 & $1 \%$ & 4 \\
\hline Varizes esofágicas & 5 & $1 \%$ & 2 \\
\hline Estenose esofágica & 1 & $0 \%$ & 2 \\
\hline Úlcera esofágica & 2 & $0 \%$ & 4 \\
\hline Xantelásma esofágico & 1 & $0 \%$ & 4 \\
\hline Nódulo submucoso & 1 & $0 \%$ & - \\
\hline Lesão elevada no esôfago & 16 & $3 \%$ & 23 \\
\hline Total & 221 & $39 \%$ & 351 \\
\hline
\end{tabular}

Fonte: Elaborado pelos autores. 
Tabela 3: Quantidade de alterações por faixa etária - Esôfago

\begin{tabular}{|c|c|c|c|c|c|c|c|}
\hline \multirow{2}{*}{ Alteração } & \multicolumn{6}{|c|}{ Faixa Etária } & \multirow{2}{*}{ Total } \\
\hline & 16-29 & $\%$ & 30-59 & $\%$ & Mais de 60 & $\%$ & \\
\hline Esofagite inespecífica & 2 & $50 \%$ & 2 & $50 \%$ & - & - & 4 \\
\hline Esofagite não erosiva & 31 & $22 \%$ & 90 & $63 \%$ & 22 & $15 \%$ & 143 \\
\hline Esofagite grau A & 23 & $16 \%$ & 101 & $72 \%$ & 17 & $12 \%$ & 141 \\
\hline Esofagite grau B & 9 & $15 \%$ & 46 & $75 \%$ & 6 & $10 \%$ & 61 \\
\hline Esofagite grau $\mathrm{C}$ & 2 & $29 \%$ & 4 & $57 \%$ & 1 & $14 \%$ & 7 \\
\hline Esofagite grau D & - & - & 4 & $80 \%$ & 1 & $20 \%$ & 5 \\
\hline Esofagite eosinofílica & - & - & 3 & $100 \%$ & - & - & 3 \\
\hline Megaesôfago & - & - & 1 & $20 \%$ & 4 & $80 \%$ & 5 \\
\hline Neoplasia de esôfago & - & - & - & - & - & - & - \\
\hline Monilíase esofágica grau I & 2 & $11 \%$ & 15 & $79 \%$ & 2 & $11 \%$ & 19 \\
\hline Monilíase esofágica grau II & - & - & 1 & $100 \%$ & - & - & 1 \\
\hline Monilíase esofágica grau III & - & - & - & - & - & - & 0 \\
\hline Monilíase esofágica grau IV & - & - & - & - & - & - & 0 \\
\hline Hérnia de hiato & 7 & $8 \%$ & 60 & $65 \%$ & 26 & $28 \%$ & 93 \\
\hline Esôfago de barret curto & 1 & $5 \%$ & 18 & $82 \%$ & 3 & $14 \%$ & 22 \\
\hline Esôfago de barret longo & 3 & $43 \%$ & 3 & $43 \%$ & 1 & $14 \%$ & 7 \\
\hline Varizes esofágicas & - & - & 5 & $71 \%$ & 2 & $29 \%$ & 7 \\
\hline Estenose esofágica & - & - & 1 & $33 \%$ & 2 & $67 \%$ & 3 \\
\hline Úlcera esofágica & 2 & $33 \%$ & 4 & $67 \%$ & - & - & 6 \\
\hline Xantelásma esofágico & - & - & 4 & $80 \%$ & 1 & $20 \%$ & 5 \\
\hline Nódulo submucoso & - & - & 1 & $100 \%$ & - & - & 1 \\
\hline Lesão elevada no esôfago & 4 & $10 \%$ & 30 & $77 \%$ & 5 & $13 \%$ & 39 \\
\hline Total & 86 & $15 \%$ & 393 & $69 \%$ & 93 & $16 \%$ & 572 \\
\hline
\end{tabular}

Fonte: Elaborado pelos autores.

Em relação ao estômago, das patologias ou achados encontrados, foi visto que $38 \%$ dos pacientes apresentavam gastrite de grau moderado, seguido de $24 \%$ gastrite de grau leve e $15 \%$ gastrite de grau severo. Embora a amostra apresente casos de lesões deprimidas gástricas e neoplasia gástrica, estes foram percentualmente insignificantes diante da totalidade amostral. Foram encontrados apenas dois casos de neoplasia gástrica do tipo Bormann 1, dois casos do tipo Bormann 2 e três casos de Bormann 3, nenhum de Bormann 4 e dois casos de adenocarcinoma. (Tabela 4). 
Research, Society and Development, v. 10, n. 13, e567101321705, 2021

(CC BY 4.0) | ISSN 2525-3409 | DOI: http://dx.doi.org/10.33448/rsd-v10i13.21705

Tabela 4: Quantidade de alterações - Estômago

\begin{tabular}{l|l|l}
\hline Alteração & $\mathbf{N}$ & $\%$ \\
\hline Gastrite grau leve & 354 & $24 \%$ \\
\hline Gastrite grau moderado & 568 & $38 \%$ \\
\hline Gastrite grau severo & 229 & $15 \%$ \\
\hline Lesão ulcerada gástrica & 62 & $4 \%$ \\
\hline Bormann 1 & 2 & $0 \%$ \\
\hline Bormann 2 & 2 & $0 \%$ \\
\hline Bormann 3 & 3 & $0 \%$ \\
\hline Bormann 4 & - & - \\
\hline Gastropatia hipertensiva portal & 3 & $0 \%$ \\
\hline Telangiectasia gástrica & 13 & $1 \%$ \\
\hline Refluxo duodeno-gástrico & 58 & $4 \%$ \\
\hline Lesão submucosa gástrica & 15 & $1 \%$ \\
\hline Lesão deprimida gástrica (Adenocarcinoma) & 2 & $0 \%$ \\
\hline Lesão elevada gástrica/Pólipo & 188 & $13 \%$ \\
\hline Total & $\mathbf{1 4 9 9}$ & \\
\hline
\end{tabular}

Fonte: Elaborado pelos autores.

Quanto a patologias por faixa etária, foi visto que as gastrites foram encontradas em maior proporção na população entre 30 a 59 anos, representando de 65 até 70\% dos casos, além disso, os casos de neoplasia gástrica do tipo Bormman 3 foram todos encontrados nessa mesma faixa etária e 50\% dos casos de Bormann 2 estavam na faixa etária dos maiores de 60 anos. (Tabela 5) 
Research, Society and Development, v. 10, n. 13, e567101321705, 2021

(CC BY 4.0) | ISSN 2525-3409 | DOI: http://dx.doi.org/10.33448/rsd-v10i13.21705

Tabela 5: Quantidade de alterações por faixa etária - Estômago

\begin{tabular}{|c|c|c|c|c|c|c|c|}
\hline \multirow{2}{*}{ Alteração } & \multicolumn{6}{|c|}{ Faixa Etária } & \multirow{2}{*}{ Total } \\
\hline & $16-29$ & $\%$ & $30-59$ & $\%$ & Mais de 60 & $\%$ & \\
\hline Gastrite grau leve & 93 & $26 \%$ & 232 & $66 \%$ & 29 & $8 \%$ & 354 \\
\hline Gastrite grau moderado & 86 & $15 \%$ & 399 & $70 \%$ & 83 & $15 \%$ & 568 \\
\hline Gastrite grau severo & 41 & $18 \%$ & 148 & $65 \%$ & 40 & $17 \%$ & 229 \\
\hline Lesão ulcerada gástrica & 10 & $16 \%$ & 39 & $63 \%$ & 13 & $21 \%$ & 62 \\
\hline Bormann 1 & - & - & 1 & $50 \%$ & 1 & $50 \%$ & 2 \\
\hline Bormann 2 & 1 & $50 \%$ & - & - & 1 & $50 \%$ & 2 \\
\hline Bormann 3 & - & - & 3 & $100 \%$ & - & - & 3 \\
\hline Bormann 4 & - & - & - & - & - & - & 0 \\
\hline Gastropatia hipertensiva portal & - & - & 3 & $100 \%$ & - & - & 3 \\
\hline Telangiectasia gástrica & 2 & $15 \%$ & 9 & $69 \%$ & 2 & $15 \%$ & 13 \\
\hline Refluxo duodeno-gástrico & 10 & $17 \%$ & 41 & $71 \%$ & 7 & $12 \%$ & 58 \\
\hline Lesão submucosa gástrica & - & - & 12 & $80 \%$ & 3 & $20 \%$ & 15 \\
\hline Lesão deprimida gástrica & - & - & 2 & $100 \%$ & - & - & 2 \\
\hline Lesão elevada/Pólipo & 28 & $15 \%$ & 124 & $66 \%$ & 36 & $19 \%$ & 188 \\
\hline Total & 271 & $18 \%$ & 1013 & $68 \%$ & 215 & $14 \%$ & 1499 \\
\hline
\end{tabular}

Fonte: Elaborado pelos autores.

Em relação ao percentual por sexo, na gastrite foi visto que houve maior número de mulheres acometidas, variando de $10 \%$ a $25 \%$, certamente devido ao maior número de participantes do sexo feminino. Quanto aos casos de neoplasia gástrica, foi observado maior prevalência do sexo feminino. (Tabela 6). 
Research, Society and Development, v. 10, n. 13, e567101321705, 2021

(CC BY 4.0) | ISSN 2525-3409 | DOI: http://dx.doi.org/10.33448/rsd-v10i13.21705

Tabela 6: Quantidade de alterações por sexo - Estômago.

\begin{tabular}{|c|c|c|c|c|c|}
\hline \multirow{2}{*}{ Alteração } & \multicolumn{4}{|c|}{ Sexo } & \multirow{2}{*}{ Total } \\
\hline & Masculino & $\%$ & Feminino & $\%$ & \\
\hline Gastrite grau leve & 88 & $6 \%$ & 266 & $18 \%$ & 354 \\
\hline Gastrite grau moderado & 190 & $13 \%$ & 378 & $25 \%$ & 568 \\
\hline Gastrite grau severo & 80 & $5 \%$ & 149 & $10 \%$ & 229 \\
\hline Lesão ulcerada gástrica & 27 & $2 \%$ & 35 & $2 \%$ & 62 \\
\hline Bormann 1 & 1 & $0 \%$ & 1 & $0 \%$ & 2 \\
\hline Bormann 2 & - & - & 2 & $0 \%$ & 2 \\
\hline Bormann 3 & 1 & $0 \%$ & 2 & $0 \%$ & 3 \\
\hline Bormann 4 & - & - & - & - & - \\
\hline Gastropatia hipertensiva portal & 3 & $0 \%$ & - & - & 3 \\
\hline Telangiectasia gástrica & 3 & $0 \%$ & 10 & $1 \%$ & 13 \\
\hline Refluxo duodeno-gástrico & 14 & $1 \%$ & 44 & $3 \%$ & 58 \\
\hline Lesão submucosa gástrica & 6 & $0 \%$ & 9 & $1 \%$ & 15 \\
\hline Lesão deprimida gástrica & 1 & $0 \%$ & 1 & $0 \%$ & 2 \\
\hline Lesão elevada/Pólipo & 51 & $3 \%$ & 137 & $9 \%$ & 188 \\
\hline Total & 465 & $31 \%$ & 1034 & $69 \%$ & 1499 \\
\hline
\end{tabular}

Fonte: Elaborado pelos autores.

Quanto ao duodeno, foi encontrado que 30\% dos laudos apresentavam linfangiectasia idiopática, em seguida duodenite de grau leve (25\%) e duodenite de grau moderado (10\%). Em relação ao sexo, todas as patologias foram encontradas com maior proporção no sexo feminino e na faixa etária de 30 a 59 anos. (Tabela 7, tabela 8 e tabela 9). 
Tabela 7: Quantidade de alterações - Duodeno.

\begin{tabular}{l|l|l}
\hline Alteração & $\mathbf{N}$ & $\mathbf{\%}$ \\
\hline Lifangiectasia idiopática & 81 & $30 \%$ \\
\hline Duodenite grau leve & 65 & $25 \%$ \\
\hline Duodenite grau moderado & 27 & $10 \%$ \\
\hline Duodenite grau severo & 18 & $7 \%$ \\
\hline Duodenite inespecífica & 24 & $9 \%$ \\
\hline Lesão elevada/Pólipo & 22 & $8 \%$ \\
\hline Úlcera duodenal & 16 & $6 \%$ \\
\hline Fístula duodenal & 1 & $0 \%$ \\
\hline Divertículo & 1 & $0 \%$ \\
\hline Pseudodivertículo & 9 & $5 \%$ \\
\hline Total & $\mathbf{2 6 4}$ & $\mathbf{1}$ \\
\hline
\end{tabular}

Fonte: Elaborado pelos autores.

Tabela 8: Quantidade de alterações por faixa etária - Duodeno

\begin{tabular}{c|c|c|c|c|c|c|c}
\hline \multirow{2}{*}{ Alteração } & \multicolumn{5}{|c|}{ Faixa Etária } & \multirow{2}{*}{ Total } \\
\cline { 2 - 7 } & $\mathbf{1 6 - 2 9}$ & $\mathbf{9 0 - 5 9}$ & $\mathbf{3}$ & Mais de 60 & $\mathbf{\%}$ & \\
\hline Linfangiectasia intestinal idiopática & 18 & $22 \%$ & 56 & $69 \%$ & 7 & $9 \%$ & 81 \\
\hline Duodenite grau leve & 7 & $11 \%$ & 43 & $66 \%$ & 15 & $23 \%$ & 65 \\
\hline Duodenite grau moderado & 5 & $19 \%$ & 15 & $56 \%$ & 7 & $26 \%$ & 27 \\
\hline Duodenite grau severo & 2 & $11 \%$ & 12 & $67 \%$ & 4 & $22 \%$ & 18 \\
\hline Duodenite inespecífica & 4 & $17 \%$ & 12 & $50 \%$ & 8 & $33 \%$ & 24 \\
\hline Lesão elevada/Pólipo & 2 & $9 \%$ & 14 & $64 \%$ & 6 & $27 \%$ & 22 \\
\hline Úlcera duodenal & 1 & $6 \%$ & 12 & $75 \%$ & 3 & $19 \%$ & 16 \\
\hline Fístula duodenal & - & - & - & - & 1 & $100 \%$ & 1 \\
\hline Divertículo duodenal & - & - & - & - & 1 & $100 \%$ & 1 \\
\hline Pseudodivertículo duodenal & 1 & $11 \%$ & 4 & $44 \%$ & 4 & $44 \%$ & 9 \\
\hline Total & $\mathbf{4 0}$ & $\mathbf{1 5 \%}$ & $\mathbf{1 6 8}$ & $\mathbf{6 4 \%}$ & $\mathbf{5 6}$ & $\mathbf{2 1 \%}$ & $\mathbf{2 6 4}$ \\
\hline
\end{tabular}

Fonte: Elaborado pelos autores. 
Research, Society and Development, v. 10, n. 13, e567101321705, 2021

(CC BY 4.0) | ISSN 2525-3409 | DOI: http://dx.doi.org/10.33448/rsd-v10i13.21705

Tabela 9: Quantidade de alterações por sexo - Duodeno

\begin{tabular}{|c|c|c|c|c|c|}
\hline \multirow{2}{*}{ Alteração } & \multicolumn{4}{|l|}{ Sexo } & \multirow[t]{2}{*}{ Total } \\
\hline & Masculino & $\%$ & Feminino & $\%$ & \\
\hline Linfangiectasia intestinal idiopática & 25 & $9 \%$ & 56 & $21 \%$ & 81 \\
\hline Duodenite grau leve & 26 & $10 \%$ & 39 & $15 \%$ & 65 \\
\hline Duodenite grau moderado & 11 & $4 \%$ & 16 & $6 \%$ & 27 \\
\hline Duodenite grau severo & 6 & $2 \%$ & 12 & $5 \%$ & 18 \\
\hline Duodenite inespecífica & 8 & $3 \%$ & 16 & $6 \%$ & 24 \\
\hline Lesão elevada/Pólipo & 6 & $2 \%$ & 16 & $6 \%$ & 22 \\
\hline Úlcera duodenal & 9 & $3 \%$ & 7 & $3 \%$ & 16 \\
\hline Fístula duodenal & 1 & $0 \%$ & - & - & 1 \\
\hline Divertículo duodenal & 1 & $0 \%$ & - & - & 1 \\
\hline Pseudodivertículo duodenal & 4 & $2 \%$ & 5 & $2 \%$ & 9 \\
\hline Total & 97 & $37 \%$ & 167 & $63 \%$ & 264 \\
\hline
\end{tabular}

Fonte: Elaborado pelos autores.

No que diz respeito aos pacientes submetidos a algum tipo de cirurgia digestiva, foi encontrado um baixo percentual, correspondente a 2,28\% dos laudos analisados, sendo o sexo masculino com maior percentual de cirurgias (59\%) e a cirurgia de antirrefluxo sendo a mais realizada e em pacientes maiores de 60 anos. (Tabela 10).

Tabela 10: Quantidade de cirurgias por sexo

\begin{tabular}{c|c|c|c|c|c}
\hline \multirow{2}{*}{ Exames } & \multicolumn{4}{c|}{ Sexo } & \multirow{2}{*}{ Total } \\
\cline { 2 - 5 } & Masculino & $\%$ & Feminino & $\%$ & \\
\hline Gastrectomia total & 4 & $80 \%$ & 1 & $20 \%$ & 5 \\
\hline Gastrectomia parcial & 4 & $100 \%$ & - & - & 4 \\
\hline Bariátrica & 5 & $31 \%$ & 11 & $69 \%$ & 16 \\
\hline Esofagectomia & 2 & $100 \%$ & - & - & 2 \\
\hline Antirrefluxo & 5 & $71 \%$ & 2 & $29 \%$ & 7 \\
\hline Total & $\mathbf{2 0}$ & $\mathbf{5 9 \%}$ & $\mathbf{1 4}$ & $\mathbf{4 1 \%}$ & $\mathbf{3 4}$ \\
\hline
\end{tabular}

Fonte: Elaborado pelos autores.

Dos pacientes submetidos a cirurgia bariátrica, 27\% apresentaram esofagite não erosiva, seguido por $20 \%$ lesão elevada esofagiana. (Gráfico 3). Os pacientes que realizaram cirurgia antirrefluxo a maior parte apresentou gastrite moderada a 
severa ou duodenite leve. Além disso, 6.7\% apresentaram refluxo duodeno-gástrica mesmo após a cirurgia e 6,7\% apresentaram hérnia hiatal (Gráfico 4).

Gráfico 3: Gráfico das patologias encontradas em pacientes que realizaram bariátrica.

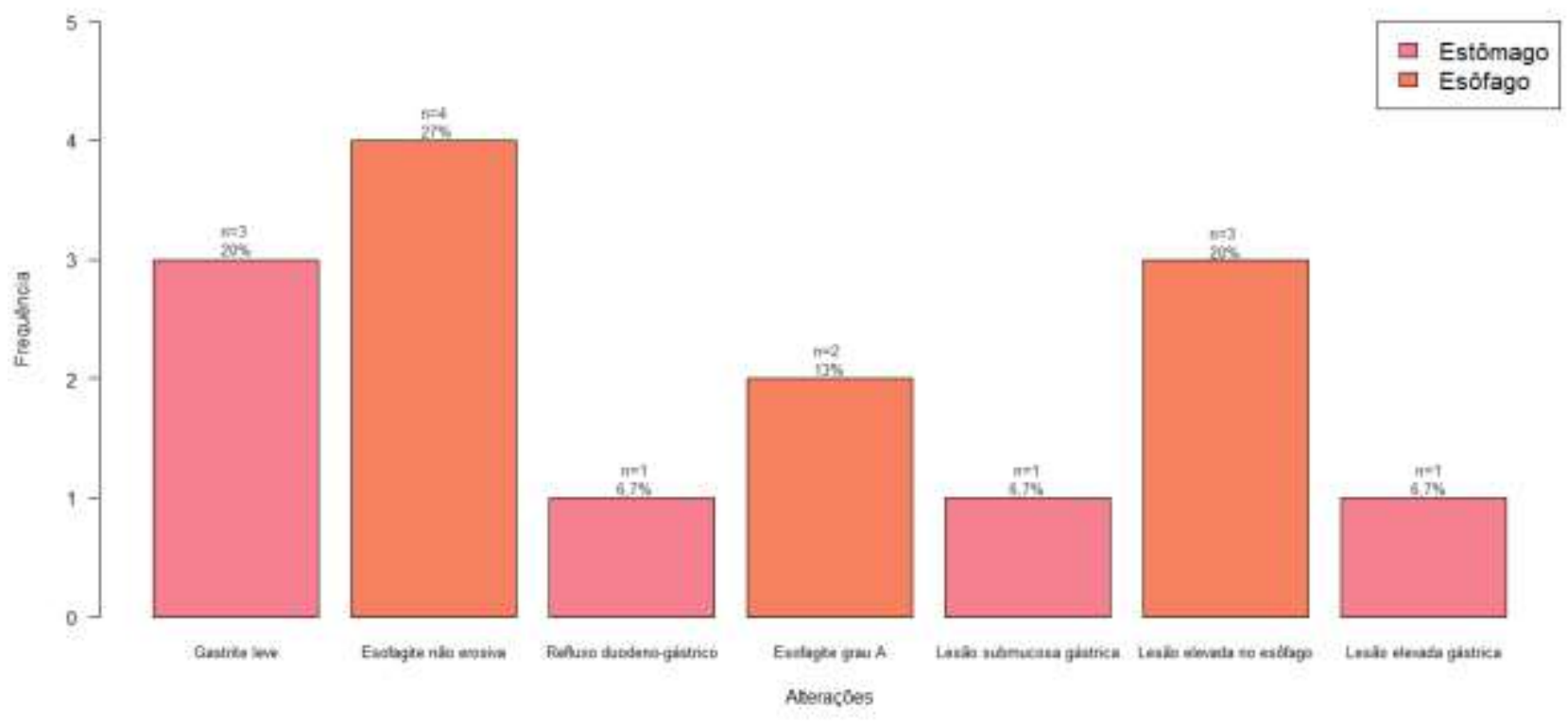

Fonte: Elaborado pelos autores.

Gráfico 4: Gráfico das patologias encontradas em pacientes que realizaram Antirrefluxo

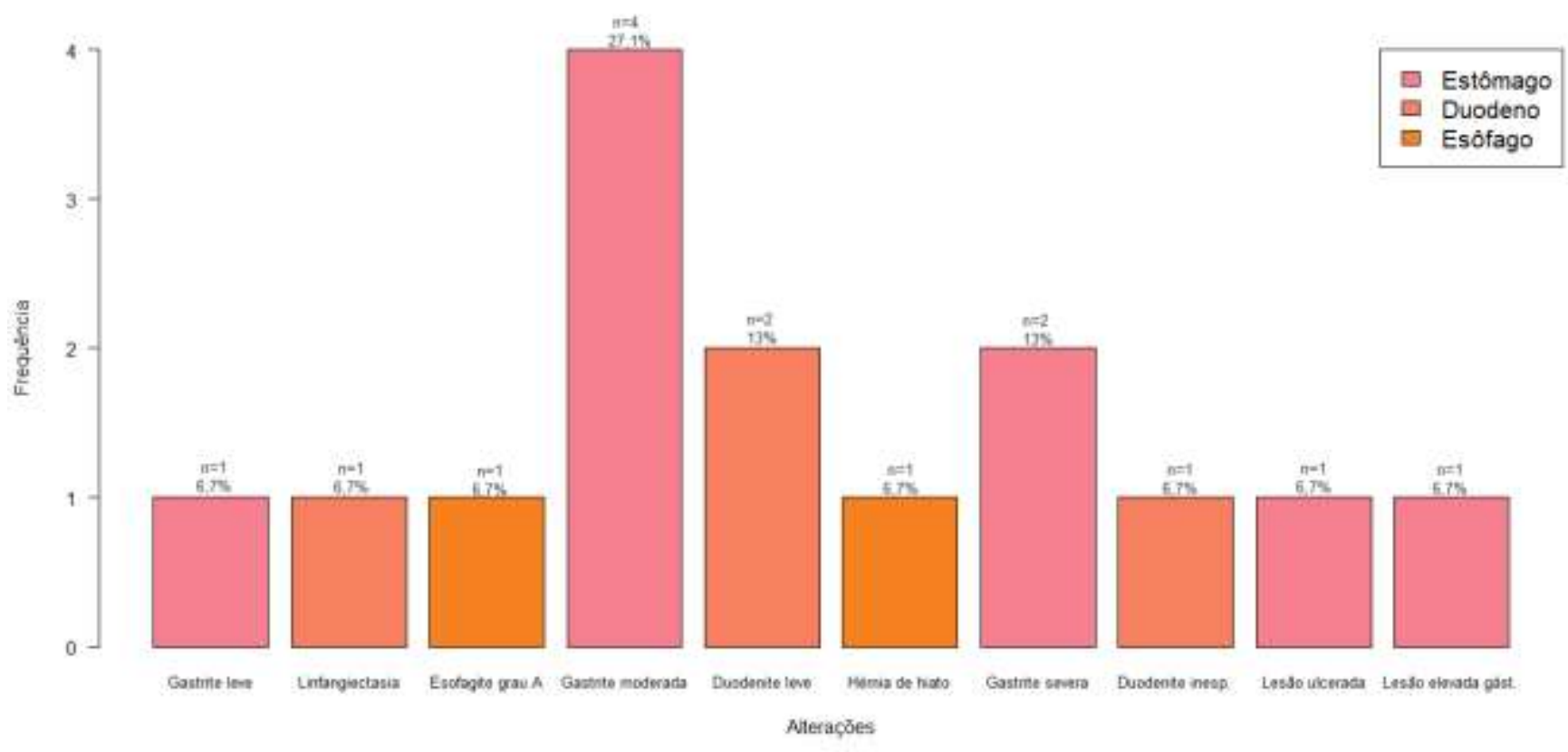

Fonte: Elaborado pelos autores. 


\section{Discussão}

Avaliando os resultados foi observado que o estômago é o órgão do trato gastrointestinal alto mais atingido, equivalendo a aproximadamente $64 \%$ dos achados, seguido do esôfago e duodeno. Dentre as alterações no estômago, a gastrite de grau moderado e leve foram os achados mais comuns, correspondendo a 38\% e 24\%. No esôfago a Esofagite não erosiva e a Esofagite grau A representaram a maioria das esofagopatias, enquanto que no duodeno prevaleceram a lifangiectasia idiopática e a duodenite grau leve.

Foi visto que estudo, realizado em um serviço privado de endoscopia digestiva localizado na cidade de Brumado, diferiu da amostra pesquisada revelando maior proporção de achados no esôfago (36,5\%), seguido de achados no estômago $(10,7 \%)$ e no duodeno $(5,0 \%)$. No presente estudo a esofagite erosiva foi o achado mais comum, representando $35,2 \%$ de todos os achados endoscópicos (Viana, 2019).

Em outro estudo, realizado na Unidade de Endoscopia do Hospital St. Dominic (SDH) de Gana, foi observado maior proporção de afecções no estômago com a gastrite sendo o achado mais frequente, equivalente a 70,4\% dos laudos endoscópicos, tal achado corrobora com os resultados obtidos neste estudo (Agyei-Nkansah, Duah, \& Alfonso, 2019).

Lima et al. (2017), em amostra estudada na comunidade de saúde de Mossoró (CSM), observaram dados semelhantes aos obtidos, revelando achados de esofagopatias em 22,7\% do total de endoscopias realizadas. Foi evidenciada também uma maior prevalência de esofagopatias no gênero masculino (27,5\%), apesar das mulheres realizarem mais EDA $(80,1 \%)$, diferindo da amostra estudada em que o gênero feminino apresentou predomínio das impressões diagnósticas de esofagopatias. Oliveira (2021) avaliou 2810 endoscopias e obteve predominância do sexo masculino com 16,68\% dos casos de esofagopatias, diferindo também do presente estudo.

Quando avaliado Esôfago de Barret, Pinto (2021) observou, dentre as endoscopias realizadas, a prevalência de 5,6\% com a faixa etária de maior predominância correspondente a maiores de 60 anos, esse dado difere dos resultados obtidos considerando que na amostrada deste estudo a patologia foi mais comum na faixa etária de 30 a 59 anos. De forma semelhante aos resultados obtidos, Anandasabapathy et. al (2007) também observaram que a idade média de diagnóstico foi de 58 anos.

No que se refere aos achados endoscópicos do estômago, Furtado \& Debiasi (2018) observou que a gastrite foi o achado predominante, enquanto Bernardo (2017) evidenciou que 1,1\% das endoscopias realizadas obtiveram diagnóstico de úlcera gástrica, de forma similar aos resultados obtidos. Entretanto, a amostra estudada por Bernardo (2017) foi composta predominantemente pelo sexo masculino e por maiores de 60 anos, diferente da amostra analisada em que predominou o sexo feminino e a faixa etária entre 30 a 59 anos.

Em relação às patologias duodenais, Lima et al. (2021) encontraram achados duodenais em 7\% dos procedimentos analisados, sendo as úlceras duodenais os achados mais comuns. Viana (2019) observou a prevalência de 5\% de duodenopatias nas endoscopias, sendo a duodenite a patologia mais frequente. De acordo com os resultados obtidos na amostra estudada, há semelhança na prevalência de achados duodenais apesar de existir uma discrepância entre as patologias mais comuns. Han et al. (2017), em estudo com 6.334 pacientes internado submetidos à EDA, observou que 7\% dos pacientes tiveram achados de lesões duodenais, a faixa etária predominante foi de 30 a maiores de 70 anos e o sexo mais comum foi o masculino. Comparando com o estudo em questão, observaram-se semelhança entre a faixa etária predominante de 30 a maiores de 60 anos e discordância em relação ao sexo mais comum.

Sobre as alterações endoscópicas em pacientes que realizaram cirurgia bariátrica, Matar et al. (2020) constatou que 37,9\% dos pacientes que realizaram a técnica cirúrgica Sleeve e 17,6\% dos que realizaram Bypass Gástrico desenvolveram algum nível de esofagite. Santana (2016), ao analisar 286 endoscopias realizadas em pacientes que foram submetidos à cirurgia bariátrica, verificou que a Esofagite não erosiva foi o achado mais comum, representando 12,6\% das alterações, corroborando com a análise da amostra avaliada em que as esofagites foram as patologias mais frequentes após o procedimento cirúrgico. 
No que tange os achados endoscópicos após realização de fundoplicatura, Martins et al. (2020) verificou a prevalência de 4\% de hernia hiatal paraesofágica, análogo ao presente trabalho. Além disso, Vicente et al. (2009) constatou que a patologia de maior incidência em pacientes após fundoplicatura foi esofagite não erosiva, correspondendo a 11,2\% dos pacientes, contrastando com os resultados obtidos que evidenciou a gastrite leve como o achado patológico mais prevalente.

\section{Conclusão}

De acordo com o estudo, foi visto que a maioria das amostras foram compostas por mulheres, sendo mais da metade das patologias encontradas no estômago. Acerca das patologias, em todos os órgãos as mais encontradas foram as de origem inflamatórias, como esofagites, gastrites e duodenites. Embora a amostra apresente casos de lesões deprimidas gástricas e neoplasia gástrica, estes foram percentualmente insignificantes diante da totalidade amostral. A faixa etária de 30-59 anos foi considerada a mais prevalente para a maiorias das patologias encontradas em esôfago, estômago e duodeno. O sexo feminino obteve o maior percentual entre as patologias mais frequentes de todo trato gastrointestinal, esse dado pode ter sido influenciado pelo estudo ser composto em sua maioria por mulheres. Quanto às cirurgias digestivas realizadas, foi encontrado na amostra um baixo percentual de pacientes que realizaram a cirurgia bariátrica ou a cirurgia anti-refluxo, no entanto, foi possível identificar uma correlação entre o procedimento cirúrgico e as patologias do trato gastrointestinal alto.

Entretanto, na literatura há divergências e convergências acerca de patologias, gênero e faixa etária prevalentes devido as diferentes características sociodemográficas e culturais, visto que os hábitos de vida e alimentares são fatores imprescindíveis para o desenvolvimento de patologias gastrointestinais.

Dessa forma, são necessários maiores estudos a fim de estabelecer melhores indicativos das variáveis sociodemográficas, utilizando como variáveis os hábitos de vida, aspectos clínicos dos participantes, além da faixa etária e do sexo, estabelecendo, dessa forma, uma relação mais precisa entre as patologias do trato gastrointestinal identificadas na EDA e as diversas variáveis clínicas e sociodemográficas.

\section{Referências}

Agyei-Nkansah, A., Duah, A., \& Alfonso, M. (2019). Indicationsandfindingsofupper gastrointestinal endoscopy in patientspresentingto a District Hospital, Ghana. The Pan African Medical Journal, 34, 82. https://doi.org/10.11604/pamj.2019.34.82.18002

Anandasabapathy, S., Jhamb, J., Davila, M., Wei, C., Morris, J., \&Bresalier, R. (2007). Clinicalandendoscopicfactorspredicthigherpathologic grades of Barrett dysplasia. Cancer, 109(4), 668-674. https://doi.org/10.1002/cncr.22451

Bernardo, G. de O. (2017). Perfil epidemiológico dos pacientes diagnosticados com úlcera gástrica elou duodenal em dois serviços de referência do extremo sul catarinense no período de um ano. 11.

Durães, E. S. M., Fabris, M. R., Faraco, A. J., Madeira, K., \& Luca, L. R. de. (2010). Análise dos achados endoscópicos em pacientes com dispepsia atendidos no Serviço de Endoscopia do Hospital São João Batista, Criciúma-SC, no período de outubro de 2008 a março de 2009. GED gastroenterol. endosc. dig, $73-$ 78.

Furtado, R. K., \&Debiasi, M. C. (2018). Perfil epidemiológico das alterações observadas em exames de endoscopias digestivas altas realizadas em um hospital particular do sul de Santa Catarina no período de 2013 a 2014. Recuperado de http://repositorio.unesc.net/handle/1/6428

Han, Y., Jung, H.-K., Chang, J. Y., Moon, C. M., Kim, S.-E., Shim, K.-N., ... Park, S. (2017). Identificationofdistinctiveclinicalsignificance in hospitalizedpatientswithendoscopic duodenal mucosal lesions. The Korean JournalofInternal Medicine, 32(5), 827-835. https://doi.org/10.3904/kjim.2015.149

Lima, Rnaldo C. A., Almeida, A. C. de, Maynarde, S. F., Barbosa, T. A., Lunguinho, V. L., \& Ferreira, L. L. R. (2017). A incidência de esofagopatias avaliadas por endoscospia digestiva alta. Revista de Ciências da Saúde Nova Esperança, 15(3), 37-45. https://doi.org/10.17695/issn.23177160.v15n3a2017p37-45

Lima, Ronaldo César Aguiar, Ferreira, L. L. R., Lima, B. D. C., Bezerra, L. T. L., Barbosa, T. A., \& Lima, T. T. L. (2021). Caracterização das endoscopias digestivas altas realizadas no município de Mossoró-RN. Revista Rede de Cuidados em Saúde, 15(1). Recuperado de http://publicacoes.unigranrio.edu.br/index.php/rcs/article/view/5326

Martins, B. C., Souza, C. S., Ruas, J. N., Furuya, C. K., Fylyk, S. N., Sakai, C. M., \& Ide, E. (2020). Endoscopic evaluation of post-fundoplication anatomy and correlation with symptomatology. ABCD. Arquivos Brasileiros de Cirurgia Digestiva (São Paulo), 33(3), e1543. https://doi.org/10.1590/0102$672020200003 \mathrm{e} 1543$ 
Research, Society and Development, v. 10, n. 13, e567101321705, 2021

(CC BY 4.0) | ISSN 2525-3409 | DOI: http://dx.doi.org/10.33448/rsd-v10i13.21705

Mata, D., Cordeiro, Y., Lima, F., Silva, T., Ceschini, T., Mendes, C., ... Ferreira, E. (2016). Helicobacter pylori e a gastrite: Um estudo retrospectivo. Revista da universidade vale do rio verde, $14,696-706$. https://doi.org/10.5892/ruvrd.v14i2.3144

Matar, R., Maselli, D., Vargas, E., Veeravich, J., Bazerbachi, F., Beran, A., ... Abu Dayyeh, B. K. (2020). EsophagitisAfterBariatricSurgery: Large Crosssectional Assessment ofanEndoscopicDatabase. ObesitySurgery, 30(1), 161-168. https://doi.org/10.1007/s11695-019-04164-0

Oliveira, F. A. B. de. (2021). Comparação dos achados esofagogastroduodenais da endoscopia de acesso direto com a endoscopia solicitada por especialistas em pacientes da região sul de Mato Grosso. Recuperado de http://repositorio.unicamp.br/jspui/handle/REPOSIP/363427

Pinto, S. H. E. S. (2021). Perfil epidemiológico de pacientes com esôfago de Barrett em um serviço de patologia de Passo Fundo-RS. Recuperado de https://rd.uffs.edu.br:8443/handle/prefix/4470]

Ramos, F. L. de P., Hora, Á. L. da, Souza, C. T. V. de, Pereira, L. O., \& Hora, D. L. da. (2016). As contribuições da epidemiologia social para a pesquisa clínica em doenças infecciosas. Revista Pan-Amazônica de Saúde, 7(esp), 221-229. https://doi.org/10.5123/S2176-62232016000500025

Rawla, P., \&Barsouk, A. (2019). Epidemiologyofgastriccancer: Global trends, riskfactorsandprevention. PrzegladGastroenterologiczny, 14(1), 26-38. https://doi.org/10.5114/pg.2018.80001

Ribeiro, H. G., Coelho, M. C. de F., \& Coelho, L. G. V. (2019). Evaluationoftheriskofgastriccancer in patientswithchronicgastritisbyhelicobacter pylori. Revista Médica de Minas Gerais, 29. https://doi.org/10.5935/2238-3182.20190034

Santana, M. F. D. (2016). Co-ocorrência dos achados endoscópicos após cirurgia bariátrica. 73.

Shaukat, A., Wang, A., Acosta, R. D., Bruining, D. H., Chandrasekhara, V., Chathadi, K. V., ... DeWitt, J. M. (2015). The role ofendoscopy in dyspepsia. Gastrointestinal Endoscopy, 82(2), 227-232. https://doi.org/10.1016/j.gie.2015.04.003

Viana, I. dos S. (2019). Endoscopia digestiva alta solicitada de forma inapropriada: Prevalência e achados clinicamente significativos (MasterThesis, Instituto de Higiene e Medicina Tropical). Instituto de Higiene e Medicina Tropical. Recuperado de https://run.unl.pt/handle/10362/98639

Vicente, A. M. B., Cardoso, S. R., Servidoni, M. de F. C. P., Meirelles, L. R. de, Silva, J. M. B., \& Costa-Pinto, E. A. L. da. (2009). Evolução clínica e endoscópica após fundoplicatura para tratamento da doença do refluxo gastroesofágico. Arquivos de Gastroenterologia, 46, 138-143. https://doi.org/10.1590/S0004-28032009000200012

Zagari, R. M., Eusebi, L. H., Rabitti, S., Cristoferi, L., Vestito, A., Pagano, N., \&Bazzoli, F. (2016). Prevalenceofupper gastrointestinal endoscopicfindings in thecommunity: A systematic review ofstudies in unselected samples ofsubjects: Endoscopicfindings in thecommunity. JournalofGastroenterologyandHepatology, 31(9), 1527-1538. https://doi.org/10.1111/jgh.13308 